\title{
Theoretical Aspects of Single-Spin Asymmetries Studies
}

\author{
S. M. Troshin, N. E. Tyurin \\ Institute for High Energy Physics, \\ 142284 Protvino, Moscow Region, Russia
}

\begin{abstract}
We consider theoretical background for experimental measurements of single-spin asymmetries. We stress the non-perturbative QCD aspects of observed asymmetries in hadronic reactions.
\end{abstract}

The very important direction in spin studies is connected with the longstanding problem of one-spin transverse asymmetries observed in violent hadron reactions [1], [2]. It is well known fact that the experimental data manifest significant one-spin transverse asymmetries.

For example, the behavior of analyzing power in hadronic scattering is rather surprising. Indeed, we could expect significant spin effects in soft reactions where the chiral $S U(3)_{L} \times S U(3)_{R}$ symmetry of QCD Lagrangian is spontaneously broken down to $S U(3)_{V}$ and therefore, there is no ground for helicity conservation. However, the observed analyzing power in the region of low transferred momenta is small and decreases with energy like an inverse power of energy.

On the other side, contrary to our QCD expectations analyzing power increases with transverse momentum when we trying to explore the region of short distances. In this kinematical region we should observe helicity conservation due to chiral invariance of QCD Lagrangian. Hadron helicity conservation in hard processes is a general principle of perturbative QCD. Violation of this principle have been observed in elastic $p p$-scattering, in two-body hadronic decays of $J / \psi$ and there are also indications for such violation in the measurements of Pauli form factor $F_{2}\left(Q^{2}\right)$.

It is evident now that new ideas and experimental data are urgently needed to study dynamics of the spin effects.

We consider possible dynamical mechanism of spin effects in elastic scattereing. In Ref. [3] we used the notions of effective chiral quark model for the description of elastic scattering at small and large angles. Different aspects 
of hadron dynamics were accounted in the framework of effective Lagrangian presented as a sum of three terms:

$$
\mathcal{L}=\mathcal{L}_{\chi}+\mathcal{L}_{I}+\mathcal{L}_{C}
$$

$\mathcal{L}_{\chi}$ is the term responsible for the spontaneous chiral symmetry breaking:

$$
\mathcal{L}_{\chi}=\bar{\psi}\left(i \partial^{\mu} \gamma_{\mu}-\hat{m}\right) \psi+\mathcal{L}_{4}+\mathcal{L}_{6}
$$

$\mathcal{L}_{4}$ is the NJL four-fermion interaction, $\mathcal{L}_{6}$ is the $U_{A}(1)$-breaking ${ }^{6}$-quark interaction. $\mathcal{L}_{\chi}$ is responsible for providing constituent quark masses and for the structure of constituent quark which includes valence quark and cloud of quark-antiquark pairs $\mathbb{4}$. $\mathcal{L}_{I}$ describes the interaction of constituent quarks and $\mathcal{L}_{C}$ - their confinement. These parts of effective interaction were taken into account at phenomenological level.

In such a model quarks appear as quasiparticles and have a complex structure. Besides its mass (consider $u$-quark as an example)

$$
m_{u}=m_{u}^{0}-g_{4}\langle u u\rangle-g_{6}\langle\bar{d} d\rangle\langle\bar{s} s\rangle
$$

the constituent quark has a finite size. We assume that the strong interaction radius of $q$-quark $r_{q}$ is determined by its mass: $r_{q}=\xi / m_{q}$. The common feature of the chiral models is the representation of a baryon as an inner core carring the baryonic charge and an outer condensate surrounding this core [5]. Following this picture it is natural to represent a hadron consisting of the inner region where valence quarks are located and the outer region filled with quark condensate [3]. Such a picture for the hadron structure implies that overlapping and interaction of peripheral condensates at hadron collision occurs at the first stage. In the overlapping region the condensates interact and as a result the massive quarks appear. Being released the part of hadron energy carried by the peripheral condensates goes for the generation of massive quarks. In another words nonlinear field couplings transform kinetic energy into internal energy of dressed quarks (see the arguments for this mechanism in [6] and references therein for the earlier works). Of course, the number of such quarks fluctuates. The average number of quarks should be proportional to convolution of the condensate distributions $D_{c}^{H}$ of colliding hadrons:

$$
N(s, b) \propto N(s) \cdot D_{c}^{A} \otimes D_{c}^{B},
$$

where the function $N(s)$ is determined by the thermodynamics of transformation of kinetic energy of interacting condenstates to the internal energy of massive quarks. To estimate the $N(s)$ it is feasible to assume that it is determined by the maximal possible energy dependence

$$
N(s) \simeq \kappa \frac{\left(1-\langle x\rangle_{q}\right) \sqrt{s}}{m_{q}},
$$


where $\langle x\rangle_{q}$ is the average fraction of energy carried by valence quarks, $m_{q}$ is the mass of constituent quark.

In the model [3] valence quarks located in the central part of a hadron are supposed to scatter in a quasi-independent way by the produced massive quarks at given impact parameter and by the other valence quarks. The averaged scattering amplitude of valence quark then may be represented in the form

$$
\left\langle f_{q}(s, b)\right\rangle=[N(s, b)+N-1]\left\langle V_{q}(b)\right\rangle,
$$

where $N=N_{1}+N_{2}$ is the total number of valence quarks in colliding hadrons, and $\left\langle V_{q}(b)\right\rangle$ is the averaged amplitude of single quark-quark scattering [3].

In this approach elastic scattering amplitude satisfies unitarity equation since it is constructed as a solution of the following equation [7]

$$
F=U+i U D F
$$

which is presented here in operator form. This relation allows one to satisfy unitarity provided the inequality $\operatorname{Im} U(s, b) \geq 0$ is fulfilled. The function $U(s, b)$ (generalized reaction matrix) [7] - the basic dynamical quantity of this approach - is chosen as a product of the averaged quark amplitudes

$$
U(s, b)=\prod_{q=1}^{N}\left\langle f_{q}(s, b)\right\rangle
$$

in accordance with assumed quasi-independent nature of valence quark scattering.

The $b$-dependence of function $\left\langle f_{q}\right\rangle$ is related to the quark formfactor behavior $\propto\left(\vec{q}^{2}+m_{q}^{2} / \xi^{2}\right)^{-2}$ and has a simple form [3] $\left\langle f_{q}\right\rangle \propto \exp \left(-m_{q} b / \xi\right)$.

Following the lines of the above considerations, the generalized reaction matrix in the pure imaginary case can be represented in the form

$$
U(s, b)=i G(N-1)^{N}\left[1+\alpha \frac{\sqrt{s}}{m_{q}}\right]^{N} \exp (-M b / \xi),
$$

where $M=\sum_{q=1}^{N} m_{q}$. This expression allows one to get the scattering amplitude as a solution of Eq. 7 which reproduces the main regularities observed in elastic scattering at small and large angles and consider spin phenomena.

For that purposes system of equations for helicity amplitudes has been solved and dynamical mechanism of quark scattering with and without helicity flip has been considered.

In particular spin of constituent quark in this model comes from the orbital moment of cloud of quark-antiquark pairs while the polarization of valence current quark and the polarization of the cloud of $\bar{q} q$ pairs compensate each other, e.g. for $z$-component of spin it means

$$
S_{q}=S_{q_{V}}+S_{\{\bar{q} q\}}+\left\langle L_{\{\bar{q} q\}}\right\rangle=1 / 2-1 / 2+1 / 2=1 / 2 .
$$


The above compensation occurs due to account of axial $U(1)_{A}$ anomaly in the framework of effective QCD. While considering the constituent quark as an extended object we can represent its spin as follows:

$$
S_{q}=\left\langle L_{\{\bar{q} q\}}\right\rangle=\omega I_{q}
$$

where $\omega$ is the angular velosity of quark matter inside the constituent quark and $I_{q}$ its moment of inertia. These notions on spin of constituent quark follows from consideration of spin in the framework of effective lagrangian approach to QCD [8], 9].

It should be noted that since spin of constituent quark is due to its orbital angular momentum the corresponding wave function should be equal to zero at $r=0$ due to centrifugal barrier. Such picture was advocated for the proton as a whole by Ralston and Pire [10].

Quark helicity flip in the model is provided by the mechanism of quark exchange where valence quark is exchanged with the quark produced under interaction of condensates. These quarks have different helicities and therefore such mechanism can lead to helicity flip quark scattering. Helicity non-flip quark scattering has another origin resulting from optical type of interaction. The above difference of these mechanisms leads to the different energy dependence and different phases of helicity flip and non-flip quark scattering amplitudes. Helicity amplitudes at hadron level in this approach as it was already mentioned are obtained as solutions of coupled system of equations which accounts unitarity in direct channel. Analyzing power in the framework of this model does not decrease with energy and has a non-zero value at $s \rightarrow \infty$. The value of analyzing power depends on the fraction of energy carried by valence quarks $k$ and the phase difference $\Delta(s) \propto(1-k) \sqrt{s} / m_{q}$ and has the following form

$$
A(s, \theta)=\frac{4 \sin \Delta(s)}{(1-k) N} f(\theta)\left[1+O\left(\frac{m_{q}^{2}}{s}\right)\right],
$$

where $N$ is the total number of valence quarks in colliding hadrons and $f(\theta)$ is the known function of scattering angle. Asymmetry here results from interference of helicity amplitudes which occurs due to resonance type of quark helicity flip scattering and continuum type of quark helicity non-flip scattering.

Analyzing power at $\sqrt{s}=2 \mathrm{TeV}$ and $-t=10(\mathrm{GeV} / \mathrm{c})^{2}$ in $p_{\uparrow} \bar{p}$-elastic scattering is predicted to be $12 \%$ while at $-t=5(\mathrm{GeV} / \mathrm{c})^{2}-A=7 \%$. Other non-perturbative models [11], [12] also predict non-zero values for analyzing power in TeV energy range.

To summarize, the measurement of analyzing power in elastic scattering at high energies will allow 
- test perturbative $\mathrm{QCD}$, mechanism, get knowledge on the region of applicability of perturbative QCD, study the transition from nonperturbative to perturbative phase of QCD;

- study of hadron structure and non-perturbative effects: spontaneous breaking of chiral symmetry and confinement.

\section{Acknowledgements}

We are grateful to N. Akchurin, M. Anselmino, A. D. Krisch and J. P. Ralston for interesting discussions, one of us (S.T.) express also his gratitude to A. D. Krisch for support of the visit to this Simposium.

\section{References}

[1] A. D. Krisch, Plenary lecture given at the 9th Intern. Symp. on High Energy Spin Physics, Bonn, Germany 1990.

[2] K. Heller, Proc. of the 10th Intern. Symp. on High Energy Spin Physics, Nagoya, 1992, p. 177.

[3] S.M. Troshin and N.E. Tyurin, , Particle World 3, No. 4, 165 (1994).

[4] K. Steininger and W. Wise, Phys. Rev. D48 1433 (1993).

[5] M.M. Islam, Z. Phys. C - Particles and Fields 53253 (1992).

[6] P. Carruthers and Minh Duong-Van, Phys. Rev. D28 130 (1983).

[7] A. A. Logunov, V. I. Savrin, N. E. Tyurin and O. A. Khrustalev, Teor. Mat. Fiz. 6157 (1971).

[8] H. Lipkin, Phys. Lett. B230 , 135 (1989);

H. Fritzsch, Phys. Lett. B256 , 75 (1991);

U. Ellwanger and B. Stech, Z. Phys. C 49 , 683 (1991).

[9] J. Ellis, S.J. Brodsky, M. Karliner, Phys. Lett. B206 , 309 (1988), J. Ellis, Y. Frishman, A. Hanany, and M. Karliner, Nucl. Phys. B382, 189 (1992).

[10] J. P. Ralston and B. Pire, High energy helicity violation in hard exclusive reactions of hadrons, Preprint Kansas 5-15-92, 1992.

[11] G. Preparata and J. Soffer. Phys.Lett. 86B , 304 (1979);

C.Bourelly and J.Soffer. Phys.Rev. D35 , 145 (1987). 
[12] M. Anselmino, P.Kroll and B.Pire, Z.Phys. C36 , 89 (1988). 